\title{
面向虚拟介入手术的电磁力反馈模型的设计与仿真
}

\author{
赵俭辉, 林远轩, 袁志勇 ${ }^{*}$ \\ (武汉大学计算机学院 武汉 430072) \\ (zhiyongyuan@whu.edu.cn)
}

\begin{abstract}
摘 要: 为满足虚拟血管介人手术中力触觉反馈的实时性和精准性, 采用磁悬浮原理设计一种力反馈模型生成虚拟 介人手术中的周向旋转力触觉. 通过分析通电线圈磁场特性和介人手术操作模式, 首先设计电磁线圈阵列的拓扑结 构和手术器械模型; 随后针对线圈阵列提出最优电流分配策略并进行仿真验证; 最后通过模型融合方法构建力矩-电 流预测模型. 实验结果表明, 该模型能以 $3 \%$ 的误差、 $40 \mathrm{~Hz}$ 的频率预测出电流，结合最优电流分配策略可对介人手术 中的关键力反馈进行实时、精确还原.
\end{abstract}

关键词：虚拟介人手术; 周向旋转力反馈; 电流分配策略; 模型融合

中图法分类号: TP391.41 DOI: 10.3724/SP.J.1089.2021.18703

\section{Designing and Simulation of Electromagnetic Force Feedback Model Focusing on Virtual Interventional Surgery}

\author{
Zhao Jianhui, Lin Yuanxuan, and Yuan Zhiyong* \\ (School of Computer Science, Wuhan University, Wuhan 430072)
}

\begin{abstract}
In order to generate the haptic force real-timely and accurately in virtual vascular intervention, an electromagnetic force feedback model is designed based on magnetic levitation principle to create the circumferential rotation haptic force. By analyzing the magnetic field characteristics of the electrified coil and the operation pattern of the interventional surgery, firstly the topological structure of the electromagnetic coil array and the surgical instrument model are proposed. Then the optimal current allocation strategy for the coil array is suggested and verified by simulation. Finally, the torque-current prediction model is established by utilizing the model fusion method. The experiments show that the proposed model can calculate the current with $3 \%$ error and at a frequency of about $40 \mathrm{~Hz}$. Combined with the optimal current allocation strategy, the crucial force feedback can be realized real-timely and accurately.
\end{abstract}

Key words: virtual interventional surgery; circumferential rotation force feedback; current allocation strategy; model fusion

介人手术作为一种新兴的治疗方式，以其适 应证广、微创、疗效确切、并发症小等特点, 在多 种重大疾病治疗中展现出很高的应用价值 ${ }^{[1]}$. 介人
手术通过医学影像设备的引导, 将特制的导管、导 丝等精密器械引人人体, 对体内病态进行诊断和 局部治疗. 医生对于导丝导管的控制能力直接关

收稿日期：2020-10-09; 修回日期：2021-01-12. 基金项目：国家自然科学基金(62073248). 赵俭辉(1975一), 男，博士，副教授， 硕士生导师, 主要研究方向为计算机图形图像处理、人机工程、智能人机交互、物联网技术; 林远轩(1997一), 男, 硕士, 主要研究方 向为计算机仿真、虚拟现实与人机交互; 袁志勇(1963-), 男, 博士, 教授, 博士生导师, CCF 高级会员, CCF 虚拟现实与可视化专委 会委员, 论文通讯作者, 主要研究方向为虚拟现实、智能人机交互、机器学习、嵌人式系统与物联网技术. 
系手术成败，然而在实际的手术操控方式下，医生 需要不断地尝试与纠错才能达到满足临床需要的 定位精度，手术时间长达 $157 \pm 81 \mathrm{~min}$ ，导致患者严重 的术后并发症以及对医生产生较大的辐射伤害 ${ }^{[2-3]}$.

虚拟介人手术系统使医生能进行针对性的术 前训练，加快手术过程，从而减少对手术双方的伤 害. 在虚拟血管介人手术系统中，用户通过操作与 导丝导管高度相似的手术器械与虚拟血管场景交 互，从而获得视觉、触觉的多感知反馈，高沉浸感 地体验介人手术进行过程. 虚拟介人手术系统的 难点在于实时精确地产生介人手术操作过程中的 反馈力，即导管轴向的推拉反馈力和导丝捻拧过 程中的周向旋转反馈力.

2000 年, 美国 CIMIT 研究中心首先开发出一 套完整的心血管介人手术训练设备 $\mathrm{ICTS}^{[4]}$, 基本 满足虚拟介人手术的训练要求, 但在力反馈精度 上有所欠缺; 瑞典 Mentice 公司和美国 Simbionix 先后推出了各具特点的设备, 这一系列设备为医 生手术训练提供了不错的选择 ${ }^{[5-6]} ; 2012$ 年, $\mathrm{Li}$ 等 ${ }^{[7]}$ 设计了一套导管手术培训系统, 并对真实血管建 模和可形变手术器械建模进行了相关的研究; 2018 年, Omisore 等 ${ }^{[8]}$ 对介人手术中的导丝导管器械进 行了模拟仿真，设计了一套虚拟手术系统；2019 年, Guo 等 ${ }^{[9]}$ 研究机器人介人手术系统中的虚拟力 反馈. 但是, 上述研究中力反馈模块大都是由接触 式的机械滑轮设计而成的. 随着磁悬浮技术的成 熟, 其诸多优越性逐渐体现, 如精密运动控制和低 能耗，除此之外，还消除了其他驱动方法中的摩擦 和动态非线性磁滞等 ${ }^{[10]}$. 近 5 年来, 本课题组运用 电磁式触觉力反馈技术和视触觉协同方法在虚拟 肾脏等器官组织的交互操作中获得了较好的沉浸 感 ${ }^{[1]}$, 其侧重点是针对虚拟组织的刚度感知. 目 前, 针对较为复杂的虚拟介人手术系统中的电磁 力反馈还缺乏系统的研究, 其技术难点在于高还 原度地复现手术操作方式和实时精确地产生手术 过程中的力反馈. 机械滑轮式力反馈技术虽然能 够实现虚拟介人手术中周向旋转力反馈的基本功 能, 但机械导轨的存在, 在较大程度上限制了操作 的自由性，与实际介人手术实施情况差距较大. 电 磁力反馈技术没有操作自由性的限制, 但目前尚 未见到使用无接触式的电磁力反馈技术实现周向 旋转反馈力的报道.

本文通过通电线圈阵列磁场特性和分析血管 介人手术操作模式，设计一种用于生成介人手术 中周向旋转反馈的电磁力模型, 其组成如下:
（1）产生对称分布磁场的 4 线圈拓扑结构模型 和配对的多自由度手术器械模型, 高度还原介人 手术的操作模式并提高术前训练效果;

（2）简洁高效的工作机制. 根据电磁力反馈模 型的设计提出基本运行原则和最优电流分配策略, 有效地降低系统能耗;

（3）快速精确的电流计算模型. 通过模型融合 方法构建力矩-电流的回归预测模型, 代替三维解 析法进行电流计算, 实现力反馈实时精确地生成, 获得较高的沉浸感.

利用计算机仿真技术对本文模型进行可行性 验证，设计实验对电流计算模型进行性能测试. 实 验结果表明, 本文模型能以 $3 \%$ 的误差、高于 $40 \mathrm{~Hz}$ 的频率进行电流计算, 并对介人手术中关键反馈 力进行实时精确还原.

\section{1 电磁力反馈模型与工作机制的设计}

当向电磁线圈中通人精确可控的驱动电流时, 目标操作空间中会产生可调控电磁场，此时带有永 磁体的手术器械在操作范围内会与电磁场相互作用 产生电磁力进而获得力触觉感知. 靠单一通电线圈 激发的磁场能实现的力触觉反馈往往较为简单, 潜 在自由度较低, 在面对复杂多自由度感知手术时, 需要设计具有特定拓扑结构的线圈阵列 ${ }^{[12-13]}$, 以获 得针对性较强的电磁力触觉. 在介人手术过程中, 操作者能感受到的主要的力反馈就是导管轴向的 推拉力和导丝周向的旋转力, 如图 1 所示. 前者力 反馈获取模式已较为成熟, 使用单线圈或 3 线圈拓 扑结构 ${ }^{[14]}$ 都能很好地产生该力反馈; 而基于磁悬 浮的周向旋转力还没有可行的研究, 因此, 本文重 点研究用于产生介人手术中导丝周向旋转力反馈 的电磁力模型.



图 1 介人手术中导丝导管力反馈示意图

\section{1 电磁力反馈模型的设计}

还原操作环境是增强虚拟手术沉浸感的一大 
措施，介人手术的操作空间基本为一个圆筒状区 域, 医生不需要进行太多横向移动, 只需要向圆筒 中递进导管和用手指拧转导丝. 因为自然界不存 在单极磁体，所以若要使两极永磁体在操作空间 中绕轴旋转, 则需要高度对称的外部电磁线圈阵 列拓扑结构.

受电动机定子转子工作原理 ${ }^{[15]}$ 的启发, 本文 设计了如图 2 所示的电磁线圈拓扑结构, 4 个完全 相同的电磁线圈摆放在同一平面上，各个线圈中 距离较近的底面的圆心依次相连会组成一个正方 形，正方形中心为 2 对互为对角线线圈的底面圆心 连线交点. 此结构具有高度对称性和灵活性，可以 根据特定情形下的力反馈要求调控任意组合的电 磁线圈中的电流.



图 2 用于生成周向旋转反馈力的电磁线圈拓扑结构

相应的手术器械模型如图 3 所示. 为了得到更 精确的视觉定位和更逼真的力触觉效果，将实际 介人手术中使用的导丝导管在本文中整合为一根 刚性竹蜻蜓状操作杆．虽然真实导丝导管由非刚 性材料制作，但相对于血管等体内环境的强度，可 认为导丝导管在手术进行过程中与血管接触部分 不会发生明显形变. 操作杆尖端小型永磁体在与 单线圈或 3 线圈激发的磁场交互时, 能生成操作杆 轴向上的反馈力; 磁化面为 2 个底面(矩形永磁体 由于制作工艺的限制无法在 2 个细窄面形成磁极) 的长筒形永磁体, 能提供手术器械周向自由度, 与 图 2 中线圈阵列激发的特定磁场交互，生成周向旋 转反馈力.



图 3 用于生成介人手术关键反馈力的手术器械模型

\section{2 电磁力反馈模型的工作机制设计}

\subsection{1 电磁力反馈模型的工作流程}

整个虚拟介人手术系统的工作流程如图 4 所 示. 当操作者控制手术器械到图 5a 所示的位置, 即长筒形永磁体的体中心与 $X Y Z$ 坐标系原点重合 时，假设虚拟场景中的血管与手术器械交互产生 形变, 且该形变效果指示手术器械应当有绕 $Z$ 轴 逆时针旋转的趋势, 则嵌人式端会根据手术器械 与虚拟血管模型的交互程度，快速地控制底层驱 动电路给相应线圈通对应大小的激励电流，从而 产生实时精确的逆时针方向的旋转力反馈, 辅助 虚拟介人手术的进行.

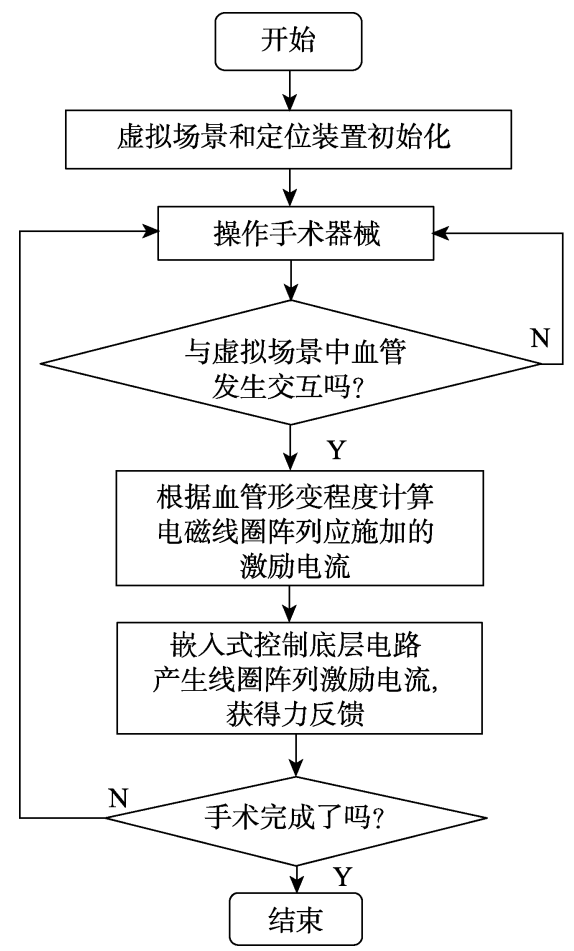

图 4 虚拟介人手术操作流程

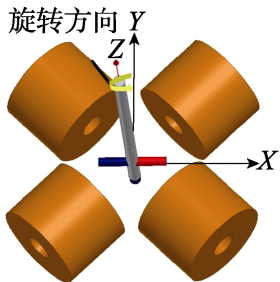

a. 工作示意图

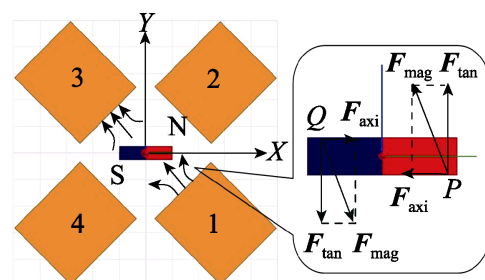

b. $X Y$ 平面切面示意图
图 5 电磁力触觉反馈模型工作示意图

\subsection{2 电磁力反馈模型的基本运行原则}

通过对刚体绕定轴旋转的运动原理分析, 操 作者要通过手术器械感受到周向旋转反馈力, 需 要满足 2 个前提条件. 
（1）长筒形永磁体整体受电磁力合力为 0 , 确 保永磁体不会产生除绕轴周向旋转以外的运动, 从而保证周向旋转力触觉的纯粹性.

(2) 长筒形永磁体绕定轴 $Z$ 轴旋转力矩不为 0 且数值较为可观, 从而保证周向旋转力触觉的有 效性.

为满足上述条件，结合通电线圈磁场分布特 性和 4 线圈阵列高度对称的拓扑结构, 针对本文设 计的模型提出以下基本运行原则：互为对角线位 置的线圈对必须给予大小方向相同的激励电流.

当向线圈对 1 和 3 通大小、方向相同的驱动电 流时, 如图 5b 所示. 假设此时手术器械受到逆时 针方向旋转的周向反馈力, 该图展示的为 $X Y$ 平面 截面图，根据通电线圈磁场分布特性，理论上，在 长筒形永磁体周围产生磁场的磁感线应如图 $5 \mathrm{~b}$ 所 示. 对长筒形永磁体 $\mathrm{N}$ 极上任意一点 $P$ 和该点关 于坐标原点对称的位于 $\mathrm{S}$ 极上的点 $Q$, 作用在点 $P$ 和点 $Q$ 上的磁感应强度大小方向都应该完全相 同, 但 $P$ 和 $Q$ 极性相反, 故点 $P$ 和 $Q$ 电磁力在宏 观上相互抵消，因此整个长筒形永磁体电磁力合 力为 0 . 然而, 微观上电磁场确实对每个小磁矩产 生电磁力 $\boldsymbol{F}_{\mathrm{mag}}$, 通过对电磁力分解, 得到永磁体 轴体方向的力 $\boldsymbol{F}_{\mathrm{axi}}$ 与绕轴旋转切向的力 $\boldsymbol{F}_{\mathrm{tan}}$, 点 $P$ 和 $Q$ 的 $\boldsymbol{F}_{\mathrm{axi}}$ 在整个永磁体空间内会被抵消, $\boldsymbol{F}_{\mathrm{tan}}$ 则 提供了永磁体绕 $Z$ 轴逆时针旋转的力矩, 使操作 者能够感受到手术器械绕 $Z$ 轴逆时针方向旋转的 反馈力。

从电磁学与运动力学的角度分析, 在遵循基 本运行原则的情况下，设计的电磁力反馈模型能 够产生纯粹有效的介人手术中的周向旋转力反馈. 下面在该模型基本运行原则的基础上，再进一步 细化线圈阵列中各线圈电流的分配策略.

\subsection{3 电磁力反馈模型的最优电流分配策略}

根据电磁力反馈模型的设计, 并以模型的基 本运行原则为基础，本文提出最优电流分配策略， 以此产生实时精确的周向旋转力反馈. 相对应地, 该策略应满足的特性如下.

（1）高效性. 通过同样大小激励电流时，选择 产生力矩较大的线圈对作为场源.

用户操作手术器械绕 $Z$ 轴旋转时, 永磁体与 各个线圈之间的夹角发生变化, 设永磁体 $\mathrm{N}$ 极与 $X$ 轴正方向夹角为 $\theta$, 当 $\theta=0^{\circ}$ 时, 如图 $5 \mathrm{~b}$ 所示, 此时如果分别向线圈对 1 和 3 或线圈对 2 和 4 施加 大小相等的激励电流, 生成的磁场与永磁体交互
产生的力矩也是相等的, 因为其相对位置完全一 样. 但当 $\theta$ 取值为 $0^{\circ} \sim 45^{\circ}$ 时, 取 $45^{\circ}$ 极限位置进行 分析, 线圈对 2 和 4 此时产生的磁场无法在永磁体 任意极上作用产生永磁体旋转切向方向的力, 因 此无论向线圈对 2 和 4 施加多大的电流, 永磁体上 的力矩都为 0 . 因此, 根据上述特殊位置的力矩情 况作出以下假设: 在 $0^{\circ} \sim 45^{\circ}$, 如果要使永磁体产生 同样大小的力矩, 线圈对 1 和 3 所需要的电流数值 比线圈对 2 和 4 所需要的电流更小, 即线圈对 1 和 3 工作更为高效.

（2）简洁性. 分析线圈阵列拓扑结构, 利用相 对位置与绝对位置的特性将模型的整个工作区域 映射到最小对称区间中.

由线圈阵列的对称性可知, 整个永磁体旋转 范围可以分割成 8 个角度为 $45^{\circ}$ 的圆弧区域，每个 区域电流激励的磁场的数值特性是完全相同的, 因此可以进行对应区域之间的映射, 从而减少数 据的计算量. 假设整个永磁体旋转区域 $\left(0^{\circ}<\theta<\right.$ $360^{\circ}$ ) 在电流和力矩等物理参数的数值上能够等价 映射在 $0^{\circ} \sim 45^{\circ}$, 即在该等价映射下, 两者使永磁体 产生同样大小的力矩, 则所施加的激励电流数值 大小应该是相同的.

基于上述对最优电流分配策略 2 个特性的假 设, 提出以下最优电流分配策略的数学表达：对永 磁体的任意角度 $\theta$, 提出一个三元组 $(x, y, z)$, 决定 电磁线圈阵列中电流的最优分配策略, 3 个元素的 计算方法分别为

$$
\begin{gathered}
x=\left[\theta / 90^{\circ}\right] \bmod 2 \\
y=\left|45^{\circ} \times\left(\left[\theta / 45^{\circ}\right] \bmod 2\right)-\theta \bmod 45^{\circ}\right| \\
z=\left[\theta / 180^{\circ}\right]
\end{gathered}
$$

该三元组中, $x$ 决定施加电流的电磁线圈对, 当 $x=0$ 时, 表示线圈对 1 和 3 工作; 当 $x=1$ 时, 线 圈对 2 和 4 工作， $y$ 为等价映射在最小对称区间内 的夹角，其取值范围为 $0^{\circ} \sim 45^{\circ} . z$ 用来指示线圈对 中电流方向, $z=0$ 时, 表示工作线圈中序号较小 的线圈的电流方向要使线圈靠近永磁体的一端产 生的磁场以 $\mathrm{N}$ 极对准永磁体, 另外一个线圈则以 $\mathrm{S}$ 极对准永磁体; $z=1$ 时, 反之. 每个 $\theta$ 对应唯一三 元组, 即唯一最优电流分配策略.

\section{2 电磁力反馈模型的物理参数计算}

虚拟介人手术系统对反馈力的计算时间和精 度都有较高的要求, 手术过程中如果反馈力数值 
误差较大或反馈力效果过于滞后, 都会产生不当 操作从而导致血管的损伤破裂，造成不可挽回的 后果. 因此, 需要建立快速精确的反馈力数值计 算模型, 一般在物理学中描述物体绕轴旋转运动 的物理量为力矩, 即建立永磁体绕转轴旋转的力 矩与各个线圈激励电流之间的表达式; 且在误差 范围内尽量简化两者之间的关系，使计算求解过 程迅速, 满足周向旋转反馈力生成的实时性和精 确性.

\section{1 三维解析计算}

2.1.1 磁感应强度的计算

(1) 单个通电线圈在空间中激发出的磁场向 量服从 Biot-Savart 定律，即

$$
\mathrm{d} \boldsymbol{B}=\frac{\mu}{4 \pi} \frac{I \mathrm{~d} \boldsymbol{l} \times \boldsymbol{e}}{R^{2}}
$$

其中, $\mathrm{d} \boldsymbol{B}$ 为磁感应强度微元； $\mu$ 为真空磁导率; $I$ 为线圈电流大小; $\mathrm{d} l$ 为电流路径的微分; $\boldsymbol{e}$ 为电 流元指向待求场点的方向向量; $R$ 为空间位置与 电流元距离. 其积分形式为

$$
\boldsymbol{B}=\int_{L} \frac{\mu I}{4 \pi} \frac{\mathrm{d} \boldsymbol{l} \times \boldsymbol{e}}{R^{2}}
$$

根据 Biot-Savart 定律易知，当通电线圈的位 置姿态固定不变且通过的电流恒定时，其在空间 中某点产生的磁场的磁感应强度大小与通电线圈

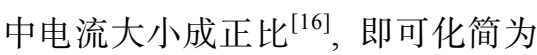

$$
B=A I
$$

其中，

$$
\boldsymbol{A}=\int_{L} \frac{\mu}{4 \pi} \frac{\mathrm{d} l \times \boldsymbol{e}}{R^{2}}
$$

（2）多个通电线圈在同一点产生的磁场的磁 感应强度符合矢量叠加原理，即在本文拓扑结构 中可分别计算 4 个线圈在同一点产生的磁场, 并最 后进行矢量叠加，即

$$
\left(\begin{array}{l}
\boldsymbol{B}_{x} \\
\boldsymbol{B}_{y} \\
\boldsymbol{B}_{z}
\end{array}\right)=\left(\begin{array}{llll}
\boldsymbol{A}_{1 x} & \boldsymbol{A}_{2 x} & \boldsymbol{A}_{3 x} & \boldsymbol{A}_{4 x} \\
\boldsymbol{A}_{1 y} & \boldsymbol{A}_{2 y} & \boldsymbol{A}_{3 y} & \boldsymbol{A}_{4 y} \\
\boldsymbol{A}_{1 z} & \boldsymbol{A}_{2 z} & \boldsymbol{A}_{3 z} & \boldsymbol{A}_{4 z}
\end{array}\right)\left(\begin{array}{c}
I_{1} \\
I_{2} \\
I_{3} \\
I_{4}
\end{array}\right)
$$

其中, 当线圈摆放位置和姿态确定时, 矩阵 $\boldsymbol{A}$ 在 解析式中就是已知量. 至此, 得到了该拓扑结构下 各个线圈电流与操作空间中任意点的磁感应强度 解析式.

\subsection{2 电磁力和力矩的计算}

根据安培分子电流假说——任何物质都有分 子电流, 即电子围绕原子核运动形成电流, 而分子 环流形成磁场，普通物质内部分子磁矩杂乱无章,
电流相互抵消，而永磁体内部分子磁矩取向趋势 一致, 宏观上有磁化电流, 其磁化电流体密度为

$$
\boldsymbol{\delta}_{v}=\nabla \times \boldsymbol{M}
$$

其中, $\nabla$ 为微分算子; $\boldsymbol{M}$ 为永磁体磁化强度, 则 电磁线圈阵列产生的磁场对永磁体作用力为

$$
\boldsymbol{F}=\iiint_{v} \boldsymbol{\delta}_{v} \times \boldsymbol{B} \mathrm{d} v=\iiint_{v}(\nabla \times \boldsymbol{M}) \times \boldsymbol{B} \mathrm{d} v
$$

对整个永磁体进行体积分, $\boldsymbol{M}$ 和 $\boldsymbol{B}$ 分别对应 永磁体上某点的磁化强度和电磁线圈阵列在该点 上产生磁场的磁感强度，在该电磁力作用下围绕 转轴 $Z$ 的力矩 ${ }^{[17]}$ 为

$$
\boldsymbol{T}=\iiint_{v} \boldsymbol{r} \times(\nabla \times \boldsymbol{M}) \times \boldsymbol{B} \mathrm{d} v
$$

其中, $r$ 为从转轴 $Z$ 指向该受力点的距离向量. 此 时只需联合式(7)(8)(11), 便能解得永磁体绕转轴旋 转力矩 $\boldsymbol{T}$ 与电磁线圈阵列中驱动电流 $\boldsymbol{I}$ 的解析式.

但是，实际应用中往往不采用这种纯解析计 算方法. 首先, 实体电磁线圈中每匝导线不是同轴 圆环回路，且漆包线和绕制工艺导致导线间有气 隙, 相关参数难以估计, 因此无法保证磁感应强 度计算的准确性; 其次, 在联立公式求解旋转力 矩或电磁线圈阵列电流的过程中大概率得不出原 解或有多个解, 实用性较差. 因此, 本文选择使 用有限元方法代替纯解析计算方法进行物理参数 求解.

\section{2 有限元计算}

有限元分析(finite element analysis, FEA)将真 实宏观的待求解物体切割成微小规则的可供内部 模块分析计算的元素单元, 随后用有限单元的未 知量去逼近无限未知量的真实系统. 由于大多数 实际问题难以得到准确解，而 FEA 不仅计算精度 较高且适用范围广，因此在计算机技术的飞速发 展下成为一种优秀强壮的数值分析方法.

本文采用 Ansoft Maxwell 电磁场 FEA 软件对 虚拟介人手术中电磁力反馈模型进行建模仿真, 向电磁线圈中分配可控电流进行磁场和力矩计算, 并通过分析求解器中电磁阵列各个线圈驱动电流 与永磁体力矩之间的对应关系, 综合验证本文模 型的可行性.

\subsection{1 模型构建}

根据第 2.1 节中电磁力、磁感应强度、电磁线 圈驱动电流之间的关系, 永磁体受到电磁力的大 小和线圈中流过电流的大小、线圈匝数、永磁体与 电磁线圈的距离和永磁体本身的材料(磁化强度)、 尺寸相关. 各参数取值的基本原则是: 在还原操作 空间的基础上, 永磁体绕轴旋转力矩范围尽可能 大. 综合考虑后, 各个参数设置如表 1 所示. 
表 1 Ansoft 软件的模型参数设置

\begin{tabular}{lc||ll}
\hline \multicolumn{1}{c|}{ 参数 } & 数值 & 参数 & 数值 \\
\hline 线圈内半径 $/ \mathrm{mm}$ & 13.5 & 永磁体半径 $/ \mathrm{mm}$ & 6 \\
线圈外半径 $/ \mathrm{mm}$ & 35 & 永磁体高度 $/ \mathrm{mm}$ & 35 \\
线圈高度 $/ \mathrm{mm}$ & 62 & 永磁体材料 & $\mathrm{NdFe} 35$ \\
线圈匝数 & 1024 & 线圈材料 & Copper \\
线圈对距离 $/ \mathrm{mm}$ & 74 & & \\
\hline
\end{tabular}

\subsection{2 仿真结果}

对电磁力反馈模型建模后，还需要设置整个 模型的气域空间、激励电流导通截面和边界条件. 根据生成磁场的分布特性和电磁线圈阵列的结构 特性，设置求解空间 $X$ 和 $Y$ 方向偏置 $50 \% ， Z$ 方 向偏置 $100 \%$, 将电磁线圈截面的一半设为电流源 端口, 采用零切向量边界条件, 即认为在求解空间 边界基本无磁场线分布. 在遵循模型基本运行原 则对线圈对 1,3 设置激励电流后，操作空间内磁场 分布如图 6 所示, 可以看出, 当互为对角线的线圈 通过同样大小激励电流时，在永磁体周围产生的 磁场数值大小分布上完全对称，方向相同.

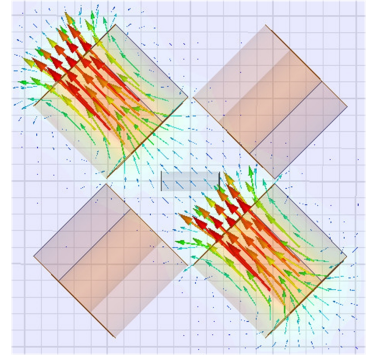

a. 磁感应强度向量图

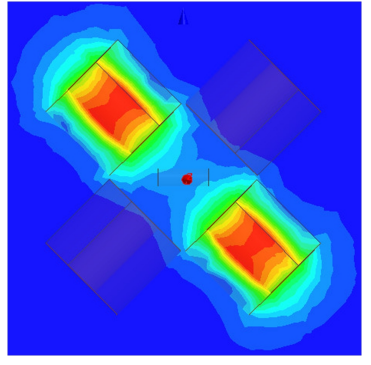

b. 磁感应强度密云图
图 $6 X Y$ 平面磁场分布情况

因此，在本文模型基本运行原则下通过仿真 求解得到的结果与模型设计时的构想一致. 随后 通过改变线圈对 1 和 3 电流大小 $C$ (电流大小为单 根导线的电流大小乘以线圈匝数, 后文中电流数 值计算均如此)分别得到永磁体力矩大小 $T$ 和电磁 力大小 $F_{\mathrm{mag}}$ 的对应关系, 如表 2 所示.

通过力矩数值计算可知，当电磁线圈中激励 电流为 $1024 \mathrm{~A}$ 时, 作用在永磁体上的旋转力约为 $0.5 \mathrm{~N}$, 对介人手术来说已经较为可观，可以再通过 加大电流获得更强的力反馈; 而此处永磁体受到的 电磁力合力数值基本可以忽略不计, 为 FEA 计算时 的误差. 所以在遵循模型基本运行原则下，上述仿 真结果显示: 该电磁力反馈模型满足手术器械中永 磁体整体合力为 0 、永磁体绕 $Z$ 轴旋转力矩数值较 为可观 2 个条件, 因此能够产生周向旋转反馈力.
表 2 线圈对 1 和 3 工作时永磁体的力矩和电磁力

\begin{tabular}{rcc}
\hline$C / \mathrm{A}$ & $T /(\mathrm{mN} \cdot \mathrm{m})$ & $F_{\mathrm{mag}} / \mathrm{N}$ \\
\hline 0.0 & 0.34941 & 0.03415 \\
204.8 & 2.15565 & 0.02969 \\
409.6 & 4.09180 & 0.01483 \\
614.4 & 5.97876 & 0.02194 \\
819.2 & 7.68081 & 0.03255 \\
1024.0 & 9.20493 & 0.04261 \\
1228.8 & 11.25274 & 0.04230 \\
1433.6 & 12.98554 & 0.02526 \\
1638.4 & 15.03375 & 0.03208 \\
1843.2 & 16.40040 & 0.03997 \\
2048.0 & 18.50768 & 0.02952 \\
\hline
\end{tabular}

然而，使用 FEA 计算的方法虽能较为精确地 得到线圈电流与永磁体力矩的计算关系, 但在实 际应用过程中 FEA 计算方法难以满足力反馈的实 时性. 可以使用此仿真模型进行离线计算获取大 量数据, 进行神经网络训练, 构建快速精确的物理 参数预测模型.

\section{3 力矩-电流预测模型的构建}

通过调整仿真软件中永磁体旋转的位置和线 圈阵列中激励电流的数值, 利用 FEA 计算永磁体 力矩数值. 收集大量离线数据训练用于回归预测 的神经网络模型, 将表现优秀的神经网络作为子 模型, 通过模型融合方法构建一个强力矩-电流预 测模型，从而快速精确地计算线圈阵列激励电流. 本文使用的子模型分别为反向传播神经网络(back propagation neural networks, BPNN)和广义回归神 经网络 (general regression neural networks, GRNN).

\section{1 数据采集与分析}

采集 Ansoft Maxwell 软件的求解器中的数据, 通过主成分分析法剔除部分无关项，组成有效合 理的数据集. 每条数据由线圈截面电流大小 $C$ 、永 磁体绕 $Z$ 轴正方向旋转的力矩大小 $T$ 和永磁体 $\mathrm{N}$ 极与 $X$ 轴正方向夹角 $\theta$ 组成. 基于该电磁力反馈 模型最优电流分配策略, 实际上只需要获得最小 对称区间，即 $\theta$ 为 $0^{\circ} \sim 45^{\circ}, C$ 为 $0 \sim 2048 \mathrm{~A}$ 的数据. 根据上述参数取值范围, 采集的总数据集由 $\theta$ 处 于 $0^{\circ} \sim 45^{\circ}$ 内均匀分布的 966 条数据构成. 数据集样 例如表 3 所示.

表 3 数据集样例

\begin{tabular}{rrr}
\hline$\theta /\left({ }^{\circ}\right)$ & $T /(\mathrm{mN} \cdot \mathrm{m})$ & $C / \mathrm{A}$ \\
\hline 5.0 & 18.400780 & 2048.0 \\
39.0 & 7.533291 & 921.6 \\
\hline
\end{tabular}




\section{2 神经网络子模型的参数设置}

\subsubsection{BPNN 参数设置}

设置 BPNN 的输人为一个由永磁体 $\mathrm{N}$ 极与 $X$ 轴正方向夹角 $\theta$ 和永磁体力矩大小 $T$ 组成的二维 向量, 输出为电磁线圈截面的激励电流大小 $C$. 根据通用近似定理 ${ }^{[18]}$, 在隐含层神经元数量足够 多的情况下，任何连续函数都可以用只含有一个 隐含层的神经网络表示, 但往往单层隐含层的 BPNN 学习到的特征较浅显, 无法比较准确地体现 输人与输出之间的内在关联. 该 BPNN 的训练集 与总数据集一致, 分析总体数据集可知, 在永磁体 绕 $Z$ 轴旋转过程中, 很难在电流固定的情况下区 分不同角度下永磁体力矩的变化规律. 因此, 本文 通过大量的针对该训练集的测试性实验，最终构 建了一个含 2 层隐含层、节点数前后分别为 7 和 8 结构的神经网络, 各训练参数如表 4 所示.

\section{表 4 BPNN 训练参数设置}

\begin{tabular}{ll}
\hline \multicolumn{1}{c}{ 训练参数 } & 数值 \\
\hline 学习率 & 0.01 \\
最大迭代次数 & 100 \\
损失函数 & 均方误差 \\
第 1 层隐含层激活函数 & Sigmoid \\
第 2 层隐含层激活函数 & ReLU \\
\hline
\end{tabular}

\subsubsection{GRNN 参数设置}

与 BPNN 不同, GRNN 的网络结构分为输人 层、模式层、求和层和输出层. 输人层节点数为样 本的特征维度; 模式层的目的是计算测试样本与 每个训练样本的高斯函数取值, 节点数为训练样 本的个数; 求和层节点数为输出样本维度 +1 , 第 1 个节点为模式层输出的算术和, 其余节点输出为 模式层输出的加权和; 输出层节点数为样本标签 维度.

对于 GRNN, 不需要像 BPNN 一样进行网络 参数权值的训练, 一旦训练集被选定, 整个网络结 构就确定下来, 可以直接进行数据预测. 因此, GRNN 需要确定的参数为采用的训练集和高斯函 数的超参数 $\delta$. 经过大量的实验, 为了平衡计算精 度与计算时间, 最后确定 GRNN 的训练集为从总 数据集中选择的 322 条数据, 其 $\delta$ 设置为 0.5 . GRNN 参数设置如表 5 所示.

\section{3 模型融合}

在回归预测场景中，模型融合方法的实质是 赋予各个子模型相应的权重，从而发挥不同子模 型各自的特点，达到互补效果，使整个经过融合处
表 5 GRNN 参数设置

\begin{tabular}{cc}
\hline 训练参数 & 数值 \\
\hline 输人层节点个数 & 2 \\
模式层节点个数 & 322 \\
求和层节点个数 & 2 \\
输出层节点个数 & 1 \\
$\delta$ & 0.5 \\
\hline
\end{tabular}

理后的预测模型在各项指标表现上优于单个子模 型. 设 $\hat{y}_{1}(\theta, t)$ 和 $\hat{y}_{2}(\theta, t)$ 分别为 BPNN 和 GRNN 的 预测值， $k_{1}$ 和 $k_{2}$ 为对应 2 个子模型的权重， $\hat{y}(\theta, t)$ 为模型融合后的力矩-电流预测模型的预测值, $\theta$ 和 $t$ 分别为子模型的 2 个输人一一永磁体 $\mathrm{N}$ 极与 $X$ 轴正方向夹角和永磁体力矩，则有

$$
\begin{gathered}
\hat{y}(\theta, t)=k_{1} \hat{y}_{1}(\theta, t)+k_{2} \hat{y}_{2}(\theta, t) \\
k_{1}+k_{2}=1
\end{gathered}
$$

整个力矩-电流预测模型的结构如图 7 所示. 当训练数据准确全面且数量足够时, BPNN 表现较 好, 各个测试集的预测效果都较为平均. GRNN 的 特点为当预测数据与训练集中某条数据特别接近 时，其预测结果与该条训练集数据基本无误差，根 据此特点, GRNN 可以作为另一个与 BPNN 特点互 补的子模型. 2 个子模型具体的权重可根据实验时 相同测试集下的表现进行估计.

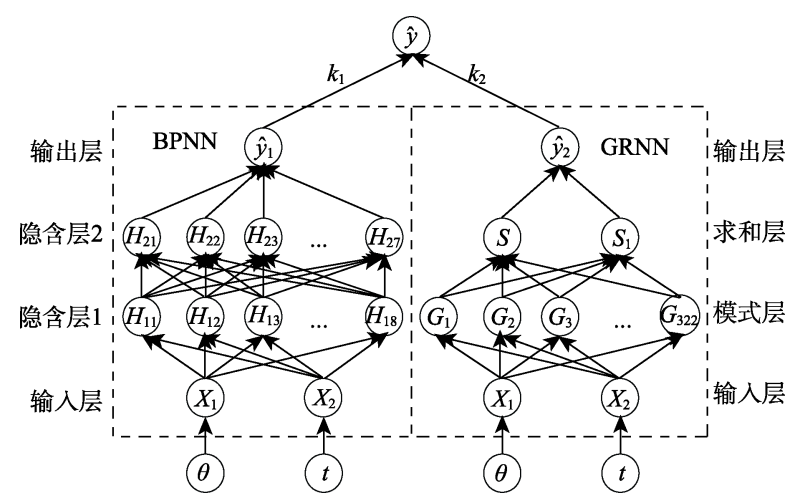

图 7 力矩-电流预测模型结构

\section{4 实验结果与分析}

\section{1 最优电流分配策略验证}

4.1.1 最优电流分配策略高效性的验证

在电磁力反馈模型的工作机制部分，本文提 出假设： $\theta$ 在 $0^{\circ} \sim 45^{\circ}$ 时, 对线圈对 1 和 3 与线圈对 2 和 4 分别施加相同大小的激励电流, 线圈对 1 和 3 产生的磁场使永磁体获得的力矩更大, 即线圈对 1 和 3 工作更为高效. 
根据电磁线圈磁场与电流的线性关系，只需验 证在某个确定数值的电流下, 线圈对 1 和 3 产生磁 场使永磁体获得的力矩均比线圈对 2 和 4 大即可. 因此，当 2 组线圈对分别都通 $2048 \mathrm{~A}$ 大小的激励电 流时, 其永磁体力矩随着角度 $\theta$ 变化, 如图 8 所示.

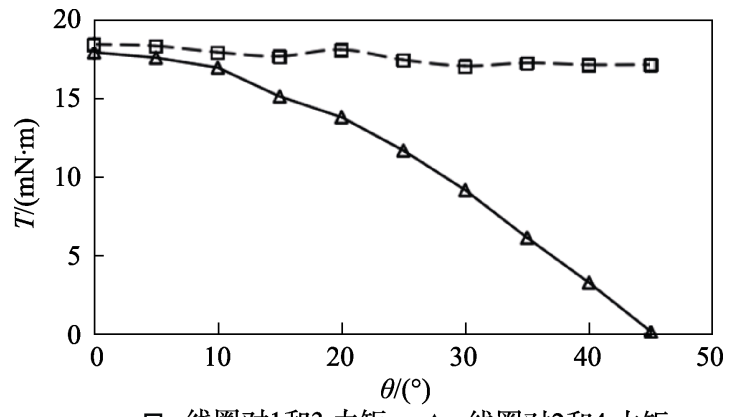

$-\boxminus 一$ 线圈对 1 和 3 力矩; $-\Delta$ 线圈对 2 和 4 力矩

图 8 线圈对 1 和 3 与线圈对 2 和 4 分别工作时 永磁体的力矩

从图 8 可以看出, 线圈对 1 和 3 中力矩随夹角 变化的幅度不大，而线圈对 2 和 4 随夹角增大而减 小, 在 $\theta=45^{\circ}$ 时趋近于 0 . 事实上，在夹角增大的 同时，永磁体整体离线圈对 1 和 3 较远，但是电磁 力方向反而越贴近切向，而线圈对 2 和 4 虽然离永 磁体越来越近, 但电磁力在切向上的分量越来越 小, 显然, 角度分量上的变化在此结构中对力矩的 影响更大. 所以当给予同样大小的激励电流时，在 $\theta$ 为 $0^{\circ} \sim 45^{\circ}$ (本文中只给出间隔为 $5^{\circ}$ 的数据) 时, 线 圈对 1 和 3 对永磁体产生的力矩比线圈对 2 和 4 产 生的力矩更大, 即线圈对 1,3 中电流对永磁体力矩 贡献更大, 以此验证最优电流分配策略的高效性.

\subsection{2 最优电流分配策略简洁性的验证}

对分布在 $0^{\circ} \sim 360^{\circ}$ 的 $\theta$ ，根据最优电流分配策 略的计算公式求出唯一对应的三元组 $(x, y, z)$, 并 且选择随机大小的激励电流作为电流源; 随后分 别求解永磁体与按照电磁力反馈模型的基本运行 原则生成的磁场交互产生的力矩和与最优电流分 配策略确定的三元组指导下产生的磁场交互产生 的力矩, 对比这 2 个数据来验证该策略简洁性. 此 处，随机选择了 8 个不同取值的夹角 $\theta$ 和对应的激 励电流 $C$, 计算相同大小激励电流下的永磁体与 磁场交互产生的力矩，结果如表 6 所示.
从表 6 可以看出, $\theta$ 在 $0^{\circ} \sim 360^{\circ}$ 取任意值, 并 通过最优电流分配策略求出三元组 $(x, y, z)$, 映射 前力矩大小 $T_{\theta}$ 与映射后按照三元组 $(x, y, z)$ 设置工 作线圈对和电流方向所求出的力矩大小 $T_{y}$ 基本相 同，说明永磁体在整个工作范围内，利用线圈阵列 的对称性可以将力矩、电流等数值等价映射在最小 对称区间内. 因此，最优电流分配策略的简洁性得 到验证.

表 $6 \theta$ 与其映射后角度下 2 个位置力矩大小比较

\begin{tabular}{rlrcc}
\hline$\theta /\left(^{\circ}\right)$ & $(x, y, z)$ & \multicolumn{1}{c}{$C / \mathrm{A}$} & $T_{\theta} /(\mathrm{mN} \cdot \mathrm{m})$ & $T_{y} /(\mathrm{mN} \cdot \mathrm{m})$ \\
\hline 122 & $(1,32,0)$ & 2048.0 & 17.515 & 17.752 \\
278 & $(1,8,1)$ & 2048.0 & 18.378 & 18.366 \\
67 & $(0,23,0)$ & 2048.0 & 18.241 & 18.515 \\
169 & $(1,11,1)$ & 1331.2 & 11.765 & 11.808 \\
204 & $(0,24,1)$ & 921.6 & 7.998 & 8.034 \\
226 & $(0,44,1)$ & 1638.4 & 13.955 & 13.752 \\
330 & $(1,30,1)$ & 512.0 & 4.373 & 4.369 \\
19 & $(0,19,0)$ & 1843.2 & 16.441 & 16.441 \\
\hline
\end{tabular}

\section{2 力矩-电流预测模型结果分析}

通过使用 Ansoft Maxwell 软件仿真求解，收集 了 15 条处于可达范围内且不与训练集中重合的数 据作为测试集, 使用训练效果较好的 BPNN 和 GRNN 分别进行电流预测, 结果如图 9 所示; 并对 2 个子模型进行泛化误差分析和模型评价，选择均 方误差(mean square error, MSE) 和平均绝对误差 (mean absolute error, MAE) 等参数作为预测模型的 评价指标, 结果如表 7 所示.



图 9 神经网络电流预测值与真实值对比

表 7 评价预测结果的相关参数

\begin{tabular}{cccccccc}
\hline 子模型 & RMSE & MAE & NMAE & 最大绝对误差 & $\begin{array}{c}\text { 最大绝对百分比 } \\
\text { 误差 }\end{array}$ & $\begin{array}{c}\text { 最小绝对误差 } \\
\text { 最小绝对百分 } \\
\text { 比误差 }\end{array}$ \\
\hline BPNN & 36.419898 & 28.433488 & 0.035320 & 66.728516 & 0.228005 & 0.097961 & 0.000100 \\
GRNN & 40.164699 & 32.002327 & 0.031851 & 84.388384 & 0.112596 & 1.645595 & 0.000811 \\
\hline
\end{tabular}


从表 7 可以看出, 最大绝对百分比误差分别为 $22.8 \%$ 和 $11.3 \%$, 追查此条数据发现, 此情况发生 在实际电流值较小的情况，绝对误差并不显著. 经 过测试, BPNN 子模型对一条数据的预测时间为 $0.009 \mathrm{~s}$ 左右, GRNN 子模型预测时间为 $0.012 \mathrm{~s}$. 总 体来说, BPNN 和 GRNN 的预测子模型基本满足实 时性和精确性.

预测数据显示, GRNN 的百分比平均绝对值相 对较优, 但 BPNN 在 RMSE 和 MAE 数值上表现较 好，充分说明了 2 个网络的特性不同甚至互补. 即 BPNN 对于预测数据的普适性较强, 表现较为稳 定; 而 GRNN 则适合于预测数据与训练样本接近 的情况, 一旦预测数据不在训练样本范围内或距 离较大时, 结果不太理想. 因此, 使用模型融合的 方法构建强预测模型，利用数理统计中的极值思 想, 求解使预测值与实际值之间偏差的方差最小 的子模型权值，最后预测模型表达式为

$$
\hat{y}(\theta, t)=0.4537 \hat{y}_{1}(\theta, t)+0.5463 \hat{y}_{2}(\theta, t)
$$

其中, $\hat{y}_{1}(\theta, t)$ 和 $\hat{y}_{2}(\theta, t)$ 分别为 BPNN 预测子模型 和 GRNN 预测子模型所对应的预测值; $\hat{y}(\theta, t)$ 为 最终强预测模型的预测值. 经过模型融合处理后 的强预测模型部分评价参数如表 8 所示, 可以看 出, 整体效果全面优于融合前的 2 个子模型, 百分 比误差约为 3\%. 经过实验测试，在普通 PC 机上运 行该强力矩-电流预测模型，能以低于 $0.025 \mathrm{~s}$ 的时 间计算出激励电流，即预测频率大于 $40 \mathrm{~Hz}$, 高于 视觉定位频率.

\section{表 8 强预测模型与子模型的评价参数比较}

\begin{tabular}{lccc}
\hline \multicolumn{1}{c}{ 模型 } & RMSE & MAE & NMAE \\
\hline BPNN & 36.419898 & 28.433488 & 0.0353203 \\
GRNN & 40.164699 & 32.002327 & 0.0318513 \\
强预测模型 & 35.172553 & 26.069875 & 0.0304050 \\
\hline
\end{tabular}

从表 8 可以看出, 本文模型对电流计算的 MAE 约为 $26 \mathrm{~A}$, 该大小的电流根据最优电流分配 策略作用在永磁体上的力矩在整个操作范围内大 概为 $0.2 \mathrm{mN} \cdot \mathrm{m}$, 换算成电磁反馈力大约为 $11 \mathrm{mN}$. Guo 等 ${ }^{[19]}$ 的主从介人式机器人的力反馈误差在 $20 \mathrm{mN}$ 以内; 张扬等 ${ }^{[20]}$ 针对心血管介人手术的力 反馈部分误差不超过 $4 \%$. 总体来说，本文提出的 力矩-电流预测模型能满足旋转力反馈生成的实时 性和精确性要求。

\section{5 结 语}

本文从基于磁悬浮的虚拟介人手术系统中关
键力反馈的实时性和精确性出发, 设计了一种电 磁线圈阵列拓扑结构和手术器械模型, 基于该模 型提出简洁高效的工作机制，利用 Ansoft Maxwell 仿真软件进行验证. 通过 FEA 方法在离线状态下 计算并获取大量仿真数据, 使用仿真数据进行多 个回归预测模型训练, 最后使用模型融合方法构 建强力矩-电流预测模型快速、精确地预测电流. 实验结果表明，该模型能以 $3 \%$ 的误差、高于 $40 \mathrm{~Hz}$ 的频率预测出电流, 再结合最优电流分配策略等 工作机制, 可对介人手术中的关键力反馈进行实 时、精确还原. 未来工作中, 会将模型实体化并进 行综合调试, 继续探究 6 线圈甚至 8 线圈等拓扑结 构下线圈阵列的优缺点; 还将考虑当永磁体在 $Z$ 方向上倾斜时各个参数的变化情况, 优化模型, 增 强其鲁棒性.

\section{参考文献(References):}

[1] Arinze N, Farber A, Levin S R, et al. Perioperative outcomes after lower extremity bypass and peripheral vascular interventions in patients with morbid obesity and superobesity[J]. Journal of Vascular Surgery, 2020, 71(2): 567-574

[2] Murali N, Ludwig J M, Nezami N, et al. Oligometastatic disease and interventional oncology: rationale and research directions[J]. The Cancer Journal, 2020, 26(2): 166-173

[3] Gummadi S, Eisenbrey J R, Lyshchik A. Contrast-enhanced ultrasonography in interventional oncology[J]. Abdominal Radiology, 2018, 43(11): 3166-3175

[4] Dawson S L, Cotin S, Meglan D, et al. Designing a computer-based simulator for interventional cardiology training $[\mathrm{J}]$. Catheterization and Cardiovascular Interventions, 2000, 51(4): 522-527

[5] Cusano C. System and a method for simulating a manual interventional operation by a user in a medical procedure: U.S. patent application 11/996,169[P]. 2006-7-19

[6] Bronstein R, Israeli S. Medical simulation device with motion detector: U.S. patent application 11/603,197[P]. 2006-11-22

[7] Li S, Guo J X, Wang Q, et al. A catheterization-training simulator based on a fast multigrid solver[J]. IEEE Computer Graphics and Applications, 2012, 32(6): 56-70

[8] Omisore O M, Han S P, Ren L X, et al. Towards characterization and adaptive compensation of backlash in a novel robotic catheter system for cardiovascular interventions[J]. IEEE Transactions on Biomedical Circuits and Systems, 2018, 12(4): 824-838

[9] Guo J, Jin X L, Guo S X, et al. A vascular interventional surgical robotic system based on force-visual feedback[J]. IEEE Sensors Journal, 2019, 19(23): 11081-11089

[10] Kim Y, Parada G A, Liu S D, et al. Ferromagnetic soft continuum robots[J]. Science Robotics, 2019, 4(33): eaax7329

[11] Tong Q Q, Yuan Z Y, Liao X Y, et al. Magnetic levitation haptic 
augmentation for virtual tissue stiffness perception[J]. IEEE Transactions on Visualization and Computer Graphics, 2018, 24(12): 3123-3136

[12] Berkelman P, Dzadovsky M. Magnet levitation and trajectory following motion control using a planar array of cylindrical coils[C] //Proceedings of the ASME 2008 Dynamic Systems and Control Conference. New York: ASME Press, 2008: 923-930

[13] Berkelman P, Dzadovsky M. Magnetic levitation over large translation and rotation ranges in all directions[J]. IEEE/ASME Transactions on Mechatronics, 2013, 18(1): 44-52

[14] Chen Erhu, Yuan Zhiyong, Yu Zejiang, et al. Modeling, simulation and implementation of electromagnetic force feedback in virtual surgery system[J]. Journal of System Simulation, 2014, 26(9): 2003-2008(in Chinese)

(陈二虎, 袁志勇, 余泽江, 等. 虚拟手术系统中电磁力反馈 建模仿真与实现[J]. 系统仿真学报, 2014, 26(9): 2003-2008)

[15] Tucci M, Sani L, Dio V D. Optimization of a novel magneto-rheological device with permanent magnets[J]. Progress in Electromagnetics Research M, 2017, 62: 175-188

[16] Zhu Weixu, Yuan Zhiyong, Tong Qianqian. Coil magnetic field characteristics analysis and coil spatial pose calculation in magnetic haptic feedback[J]. Journal of Electronic Measurement and Instrument, 2016, 30(4): 590-597(in Chinese)

(朱炜煦, 袁志勇, 童倩倩. 电磁力反馈中磁场特性分析与 线圈姿态计算 $[\mathrm{J}]$. 电子测量与仪器学报, 2016, 30(4): 590-597)

[17] Jin P, Lin H Y, Fang S H, et al. 3-D analytical linear force and rotary torque analysis of linear and rotary permanent magnet actuator[J]. IEEE Transactions on Magnetics, 2013, 49(7): 3989-3992

[18] Cybenko G. Approximation by superpositions of a sigmoidal function[J]. Mathematics of Control, Signals and Systems, 1989, 2(4): 303-314

[19] Guo J, Yu Y, Guo S X, et al. Design and performance evaluation of a novel master manipulator for the robot-assist catheter system[C]//Proceedings of the IEEE International Conference on Mechatronics and Automation. Los Alamitos: IEEE Computer Society Press, 2016: 937-942

[20] Zhang Yang, Xie Le, Shen Hao, et al. Force feedback research for the virtual cardiovascular interventional surgery[J]. Machine Design and Research, 2017, 33(4): 58-62+67(in Chinese) (张扬, 谢叻, 沈浩, 等. 心血管介人虚拟手术的力反馈研究 [J]. 机械设计与研究, 2017, 33(4): 58-62+67) 